Clair Sullivan

Andrew Staib

Stephen Ayre

Michael Daly

Renea Collins

Michae

Draheim $^{2,3}$

Richard Ashby

1 Princess Alexandr Hospital,

Brisbane, QLD.

2 Metro South Health

Brisbane, QLD.

3 University of

Queensland,

Brisbane, QLD.

clair.sullivan@ health.qld.gov.au

doi: 10.5694/mjal6.00476

\title{
Pioneering digital disruption: Australia's first integrated digital tertiary hospital
}

\section{Digital transformation has started in Australian hospitals}

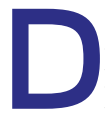

igital technology now underpins most industries; however, the health care sector (particularly in hospitals) has been slow to transform from traditional paper-based systems of care. In the United States, for example, federal legislation and financial incentives have facilitated the implementation of electronic medical records (EMRs); ${ }^{1}$ but there are only a handful of advanced EMRs in hospitals outside the US. ${ }^{2}$ The roll-out of a digital hospital includes an EMR system and other technical components, such as integrated digital vital sign monitoring and digital electrocardiogram (ECG) records. This transformation prompts revolutionary change in the way health care is delivered and monitored.

The enthusiasm for digital transformation in health has been tempered by previous failures, such as some of the unsuccessful EMR installations in the National Health Service in the United Kingdom. ${ }^{3}$ Digital health care in Australia is well established in primary care and private specialist settings, but so far it has been chequered and controversial in hospital settings, with several independent reviews previously commissioned by government and other bodies.

There are known adverse consequences and costs accompanying digital transformation of a hospital. These can include poor physician morale, increased frustration and reduced efficiency of care - particularly in the emergency department (ED) and outpatient setting. 5,6 Previous EMRs have also struggled to support traditional clinical workflows, with the American College of Physicians calling for EMRs to support the cognitive flow of physicians.

However, there are many benefits to the installation of an EMR system, which include more efficient and effective care, clinical decision support and a reduction in adverse events. ${ }^{8,9}$ There is no doubt that digital health care is the future. We describe here the challenging digital transformation of the Princess Alexandra Hospital (PAH) into the first integrated digital tertiary hospital in Australia.

\section{Digital transformation}

PAH was chosen as the exemplar site for a statewide EMR program and to become Australia's first tertiary digital hospital. It is an adult teaching hospital that delivers quaternary level care to a diverse, high acuity patient cohort. PAH has 6529 staff members, 833 overnight beds and cares for over half a million outpatients a year. The aim was to build an integrated digital hospital which provided care across the emergency, inpatient and outpatient settings. Vital sign monitoring and ECGs are digitised and delivered to the EMR via Wi-Fi for immediate viewing. Medications are the only significant component not included in this implementation and are planned for early 2017.

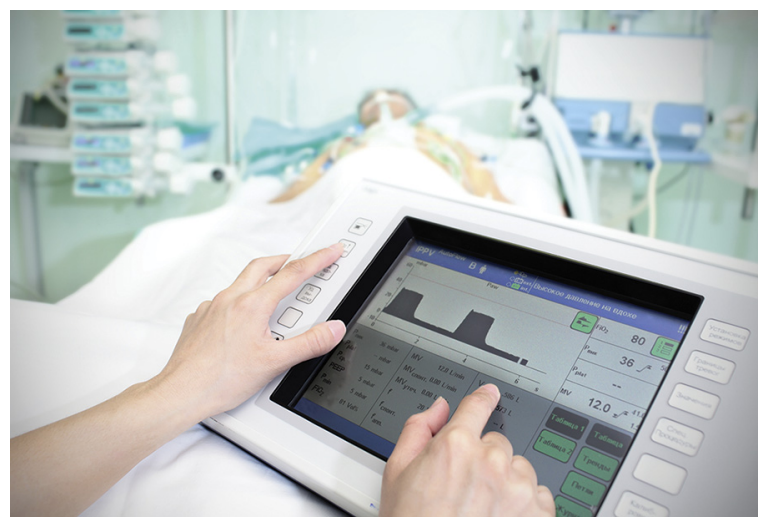

After an 18-month pre-implementation stage, the digital transformation of PAH was achieved using a big bang approach, with digital conversion occurring rapidly over 2 weeks.

\section{Clinician concerns and experiences}

It was a challenge to transform such a large, diverse organisation with a myriad of highly specialised workflows and a high acuity workload. The clinicians at our hospital had several valid concerns, the clear articulation of which influenced the course of the project.

Clinicians have had a difficult relationship with information technology (IT) projects - as evidenced by the problems affecting the Queensland health payroll. ${ }^{10}$ It became clear that a traditional IT-led approach to the digital transformation of a large tertiary hospital was unlikely to be successful. Practising clinicians - rather than IT staff with clinical backgrounds - joined the leadership team to ensure that the project remained patient and staff centred. Clinicians had to drive the project rather than act as consultants. This clinical leadership enhanced the meaningful articulation of the potential long term benefits of an EMR — particularly, the promise of a rich database of clinical information that could be used to improve the care provided.

In addition, a large amount of training was required over a short period of time. Clinicians were concerned about the adequacy of the project-prescribed training and its logistics while maintaining the existing hospital function. The preparation involved 32000 hours of training for 5384 staff. Traditional IT project-delivered web-based training alone was inadequate. Therefore, additional training, such as dress rehearsals using mock patients in clinical settings with the new technology, was organised to practise scenarios such as deteriorating patients and outpatient workflows before digitisation. This level of engagement with training required a brief reduction in elective surgical and outpatient activity. 
Clinicians were concerned that the digital transformation would slow their work speed and that it would remove existing specialised ways of undertaking complex care delivery. We customised the software to enable practice across a variety of hospital settings, from a brief fracture clinic appointment to a lengthy geriatric assessment. The executive and senior clinicians, who balanced this disruption against the long term clinical benefits, articulated an acceptance that care would be temporarily slower and less efficient.

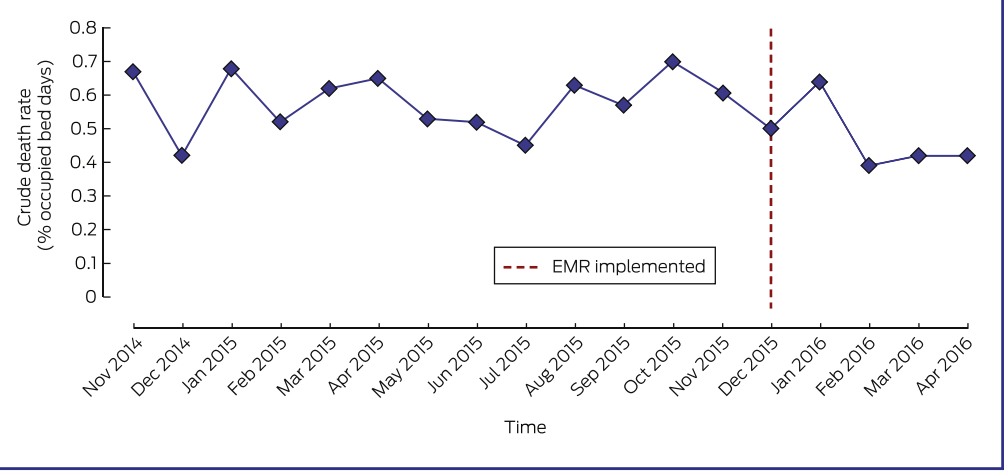

Concerns about patient safety during the digital transformation have been one of the barriers to EMR implementation. ${ }^{11}$ The EMR roll-out at $\mathrm{PAH}$ on a large and rapid scale involved a series of potential risks for our patients, including possible suboptimal design of the off the shelf systems causing errors of omission and commission; difficult integration of systems leading to errors from delays or lost information; the learning curve of the practitioners using the system; numerous changes to clinical workflows; and loss of patient focus, as attention was also directed to new technology.

In order to deal with these concerns, a patient safety team, independent of the digital hospital project, was deployed to create a patient safety watch to monitor for adverse events across a suite of pre-determined patient outcomes. The patient safety watch consisted of three components:

- patient safety officers, who were clinicians deployed on the IT help desk - on a 24 hours a day basis to monitor calls and to escalate patient safety concerns that could be hidden among the technical problems reported by staff;

- patient safety rounds, which consisted of senior clinicians walking the hospital over the initial 2-week period to assess if there were any unreported patient safety concerns; and

- patient safety data dashboard, where a dashboard, which monitored several indicators, including calls to deteriorating patients and cardiac arrest rates, was deployed with the roll-out. The mortality rate was also closely monitored.

During the initial 2-week period, the patient safety watch reported to the hospital executive implementation committee twice daily to ensure that there was strong clinical governance and that the roll-out was safe to continue and risks were being managed appropriately. It was this clinical endorsement that then allowed the IT team to progress to the next step of the roll-out. This clear, independent focus on patient safety outcomes contributed to clinician engagement and reduced anxiety.

\section{Patient outcomes during digitisation}

Although adverse events continued to occur, we did not detect any significant harm directly attributable to the digital transformation. We have monitored patient outcomes closely for the 6 months post roll-out and, when compared with the same time period the previous year to help account for seasonal variation, we found no significant difference in the number of rapid response team calls to deteriorating patients or cardiac arrests (Appendix). There were numerous anecdotes suggesting that we could expect a spike in mortality associated with a large EMR roll-out. Although there is not yet enough data for a statistically robust mortality analysis, no such mortality spike was observed (Box).

\section{Challenges of the EMR roll-out}

There still needs to be a completion of the clinical workflows with electronic prescribing. A major source of technical challenges relates to the interfaces between the new system and legacy systems, such as the patient administration system, some of which have been in service for more than 30 years.

There was a widespread disruption of normal workflows. Efficiency in the ED was reduced, with an initial $25 \%$ increase in average ED length of stay. After 6 weeks, this had begun to improve with a return to baseline at 6 months. There has been a slowing of procedural efficiency (eg, operating theatres), which continues to improve as optimisation of the software and workflows occurs. Outpatient workflow was also disrupted for some particularly large complex clinics with many new patients, such as the oncology clinic.

Retrieving meaningful clinical data for large groups of patients from our system is more challenging than expected. Significant clinical input is required to build data definitions and to extract clinically appropriate information.

\section{Benefits of the EMR roll-out}

Patient records are now readily available throughout the hospital. The digital recording of all ECGs and vital signs within the hospital has improved visibility of this information. Support for decision making has been deployed in areas such as identifying deteriorating patients. Laborious paper chart audits to record patient outcomes have been previously involved in hospital quality improvement projects. However, the digital hospital has the potential to 
provide readily available data to improve the quality and efficiency of care.

Data from the EMR will be collated to provide rich clinical information to facilitate system improvements. We have started by developing a digital dashboard that reports the details of calls to deteriorating patients in the hospital. These data enable visibility of our operations and patient outcomes in this critical area.

\section{Lessons learnt}

Establishing Australia's first tertiary digital hospital was a difficult task. Strong executive and clinical leadership was essential. Disrupted workflows and reporting structures still require refinement; nevertheless, very few staff members have expressed a wish to return to paper.

Disrupted workflows will become more efficient; however, a true return on this significant investment will be delivered when the data collected can be used to improve the quality and efficiency of health care delivery. Some data are already being provided for clinical and operational improvement, but a mature, easily accessible database will take years to establish.

Because the digital transformation of a hospital is a clinical event, patient outcomes, rather than IT project goals, need to remain the focus. Clinical leadership is essential for this process.

Acknowledgements: We thank Michael Zanco, Executive Director of Healthcare Improvement Unit at Queensland Health

Competing interests: No relevant disclosures.

Provenance: Not commissioned; externally peer reviewed.

(C) 2016 AMPCo Pty Ltd. Produced with Elsevier B.V. All rights reserved.

References are available online at www.mja.com.au. 
1 Wachter R. The digital doctor. Hype, hope and harm at the dawn of medicine's computer age. New York: McGraw Hill Education; 2015.

2 Healthcare Information Management Systems Society Analytics [website]. HiMSS Analytics. Awards and recognition 2016. Singapore: HiMSS Analytics. http://www.himssanalyticsasia.org/emram/stage7hospitals.asp (accessed Mar 2016).

3 McLoughlin I, Wilson R. Digital government at work: a social informatics perspective. Oxford: Oxford University Press; 2013.

4 Deloitte Touche Tohmatsu. NSW Health. Independent review of Cerner FirstNet. Deloitte Touche Tohmatsu; 2011 http://www.ecinsw.com.au/sites/ default/files/Deloitte\%20Report\%20FirstNet.pdf (accessed Mar 2016).

5 Sheikh A, Cornford T, Barber N, et al. Implementation and adoption of nationwide electronic health records in secondary care in England: final qualitative results from prospective national evaluation in "early adopter" hospitals. BMJ 2011; 343: d6054.

6 Agha L. The effects of health information technology on the costs and quality of medical care. J Health Econ 2014; 34: 19-30.
7 Kuhn T, Basch P, Barr M, Yackel T, Medical Informatics Committee of the American College of Physicians. Clinical documentation in the 21st century: executive summary of a policy position paper from the American College of Physicians. Ann Intern Med 2015; 162: 301-303.

8 Amarasingham R, Plantinga L, Diener-West M, et al. Clinical information technologies and inpatient outcomes: a multiple hospital study. Arch Intern Med 2009; 169: 108-114.

9 Furukawa MF, Eldridge N, Wang Y, Metersky M. Electronic health record adoption and rates of in-hospital adverse events. J Patient Saf 2016; doi: 10.1097/PTS.0000000000000257 [Epub ahead of print].

10 Chesterman RN. Queensland Health Payroll System Commission of Inquiry. Brisbane: QHPCl; 2013. http://www.healthpayrollinquiry.qld.gov.au/_data/ assets/pdf_file/0014/207203/Queensland-Health-Payroll-SystemCommission-of-Inquiry-Report-31-July-2013.pdf (accessed June 2016).

11 Singh H, Dean FS. Measuring and improving patient safety though health information technology: the Health IT Safety Framework. BMJ Qual Saf 2016; 25: 226-232. 